\title{
Bound and Total Phenolic Contents and Antioxidant Potential of Selected Sri Lankan Millet Varieties
}

\author{
N. Jayawardana, K.M.S. Wimalasiri ${ }^{1}$, G. Samarasinghe ${ }^{2}$ and T. Madhujith ${ }^{1^{*}}$ \\ Postgraduate Institute of Agriculture \\ University of Peradeniya \\ Sri Lanka
}

\begin{abstract}
Millet is a rich source of micro-nutrients and phytochemicals. However, literature on Sri Lankan millet varieties is limited. The present study was carried out to investigate the antioxidant activity (AOA), Total Phenolic Content (TPC) and Bound Phenolic Content of two varieties of finger millet (Eleusine coracana) and three varieties of foxtail millet (Setaria italica) prominently grown in Sri Lanka. The finger millet varieties tested were Wadimal Kurakkan and Bala Kurakkan whereas foxial millet varieties were Ran Thana Haal, Kaha Thana Haal and Kalu Thana Haal. AOA was screened using DPPH $(1,1$ diphenyl-2-picryl-hydrazyl) and Ferric Reducing Antioxidant Power (FRAP). Folin-Ciocalteu method was used to determine the TPC of both soluble and bound contents. According to the results, AOA showed a significant correlation with TPC while a moderate correlation was observed with FRAP and DPPH. Therefore, a distinct content of PC has contributed to the AOA in the evaluated millet varieties. Finger millet varieties showed the strongest antioxidant activities compared to the foxtail varieties in all the assays performed. The total and bound phenolic contents were observed to be higher in finger millet varieties than those in the foxtail millet varieties.
\end{abstract}

Keywords: Anti-oxidant, DPPH, finger millet, foxtail millet, FRAP, phenolic content

\section{INTRODUCTION}

The oxidative stress caused by the imbalance between antioxidant defense mechanisms and increased production of free radical is considered to be a leading cause in the development of chronic degenerative diseases (Chandrasekara et al., 2010). In the past two decades, there has been an emerging interest in phytochemicals due to their health promoting functions. Although numerous phytochemicals have been identified in plant sources, more than half remains to be unidentified in their antioxidant capacities. Therefore, it is important to explore the bioactive components of natural food crops such as fruits, vegetables, grains and spices. Grains contribute a significant supply of antioxidants which repair the damage of free radicals by donating electrons and hydrogen atoms. Epidemiological studies have demonstrated that regular consumption of whole grain cereals and their products can protect against the risk of cardiovascular diseases, type II diabetes, gastrointestinal cancers, and a range of other disorders (McKeown et al., 2002). Millet is so important because of its uniquely high content of nutrients, including impressive starch levels, very high B-vitamin

\footnotetext{
1 Department of Food Science and Technology, Faculty of Agriculture, University of Peradeniya, Sri Lanka

Plant Genetic Resources Center, Gannoruwa, Sri Lanka

* Corresponding author: madujith@yahoo.com
} 
content, as well as its high proportion of phytochemicals. In addition to antioxidant properties, polyphenols of millets, particularly finger millet, possess other health benefits such as antimicrobial, anti-inflammatory, antiviral, anticancer and antiplatelet aggregation (Hegde et al., 2005). However, the available research evidence about the antioxidant activities of local varieties of foxtail millet and finger millet in Sri Lanka is limited. The main objective of the present study was to evaluate the phenolic content of both total and bound forms present in selected local varieties of millet species grown in Sri Lanka and to evaluate the antioxidant potential of them.

\section{MATERIALS AND METHODS}

Two varieties of millets grown in Sri Lanka namely finger millet (Elusine coracana) [Bala, Wadimal] and foxtail millet (Setaria italica) [Ran, Kaha, Kalu] were obtained from Research Stations of Department of Agriculture of Sri Lanka (Plate 1). The samples were cleaned, ground to a fine powder and pooled assuring the uniform distribution and stored at $20^{\circ} \mathrm{C}$ until further analysis. Plate 1 indicates the grain of millets used for the study.

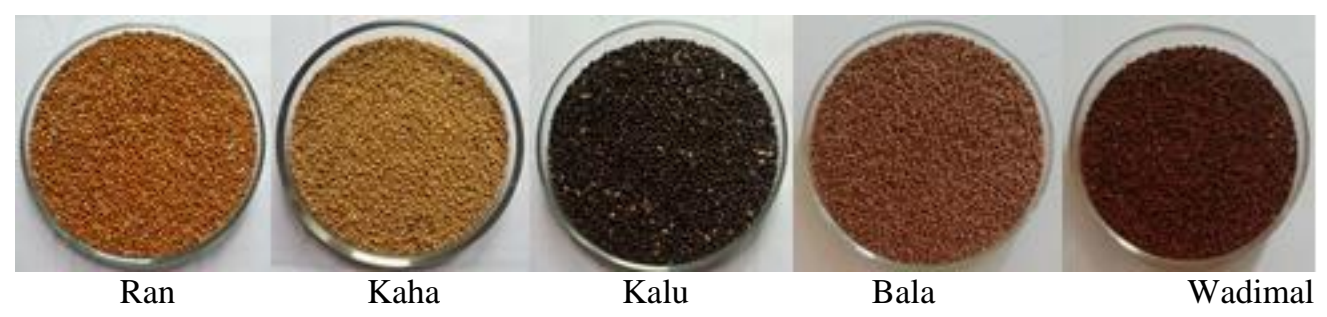

Foxtail Millets

Finger Millets

\section{Plate 1: Varieties of millets used in the study}

A sample $(0.1 \mathrm{~g})$ was ground with $7.5 \mathrm{ml}$ of distilled water and centrifuged at $2500 \mathrm{rpm}$ for $10 \mathrm{~min}$ in a centrifuge (Himac CT4D, Japan). The supernatant was filtered and the extract was appropriately diluted with distilled water before analysis. Total Phenolic Content (TPC) of the extracts was determined spectrometrically as described by Singleton et al. (1965) with slight modifications. The reaction mixture was prepared by mixing $50 \mu \mathrm{L}$ of sample extract and $105 \mu \mathrm{L}$ of $10 \%$ Folin-Ciocalteu's reagent dissolved in distilled water and $80 \mu \mathrm{L}$ of sodium carbonate $(7.5 \%$, w/v) subsequently $15 \mu \mathrm{L}$ of distilled water were added after $5 \mathrm{~min}$. The mixture was incubated for $30 \mathrm{~min}$ at room temperature and the absorbance was read at $760 \mathrm{~nm}$ using UV-Visible microplate spectrophotometer (Thermoscientific-Multiskan GO, Japan). The extraction of bound phenolic content was performed according to the procedure explained by Chandrasekara et al. (2010) with slight modifications. The resulting methanolic extracts $(0.25 \mathrm{ml})$ were mixed with $0.25 \mathrm{ml}$ of Folin-Ciocalteu's reagent in a microcentrifuge tube and mixed vigorously by vortexing. Subsequently, $0.5 \mathrm{ml}$ of $\mathrm{Na}_{2} \mathrm{CO}_{3}$ was added to neutralize the reaction mixture and diluted with $4 \mathrm{ml}$ distilled water. The tubes were allowed to incubate at room temperature for $30 \mathrm{~min}$ and centrifuged for $5 \mathrm{~min}$ at $2500 \mathrm{rpm}$. The absorbance of the resulting mixture was read at $725 \mathrm{~nm}$ using a UV Visible microplate spectrophotometer (Thermoscientific-Multiskan GO, Japan). The FRAP assay was carried out according to the procedure described by Li Fu et al. (2011) with modifications. Briefly, the freshly prepared FRAP reagent was warmed to $37^{\circ} \mathrm{C}$ in a water bath before use. Diluted 
samples $(10 \mu \mathrm{L})$ were added to $150 \mu \mathrm{L}$ of the FRAP reagent. The absorbance of the mixture was measured at $593 \mathrm{~nm}$ using a UV Visible microplate spectrophotometer (Thermoscientific-Multiskan GO, Japan) after $10 \mathrm{~min}$. Gallic acid was used as the standard. The free radical scavenging activity of the extracts was measured in terms of hydrogen donating or radical scavenging ability using the stable free radical DPPH according to the method described by Liyanage et al. (2011) with slight alterations. Briefly, the aqueous sample extracts of different concentrations were added to $100 \mu \mathrm{L}$ DPPH solution and diluted with distilled water until the total volume reached $250 \mu \mathrm{L}$. After incubating for $30 \mathrm{~min}$ in the dark at room temperature, the absorbance was read at $517 \mathrm{~nm}$ using a UV Visible microplate spectrophotometer (Thermoscientific-Multiskan GO, Japan). All the experiments were performed in triplicate and the results are expressed as mean \pm SD (standard deviation). Correlation between the phenolic contents and antioxidant activities were determined.

\section{RESULTS AND DISCUSSION}

\section{Total phenol content}

The Folin-Ciocalteu's assay was used to determine the phenolic contents on basis of the reducing activity of phenolic hydroxyl groups. The phenolic content of the extracts varied from 123.20 to $181.39 \mathrm{mg} / \mathrm{GAE} / 100 \mathrm{~g}$. Wadimal Kurakkan (finger millet) exhibited the highest phenolic content while the lowest was observed in Kaha kurakkan (foxtail millet).The results obtained from the estimation of insoluble bound forms of phenolics are illustrated in the Figure 1. The bound phenolic content of the tested millets ranged between $102.28-72.82 \mathrm{mg} / 100 \mathrm{~g} / \mathrm{GAE}$. The highest bound phenolic content was observed in Ran variety while the lowest was observed in Kaha variety of foxtail millet. In addition, the bound phenolic content showed a significant portion of polyphenolics present in all the varieties. The extraction method used in the present study would have affected the accuracy of the results since ultrasonic assisted alkaline hydrolysis was followed in this study. The bound phenolic compounds are covalently bound to cellulose, pectin and polysaccharides through ester bonds, and are difficult to be hydrolyzed (Cuevas et al., 2011). Enzymatic, thermal and alkaline hydrolysis methods have been used to extract bound phenolic compounds from different sources, with alkaline hydrolysis being the most common. The method of extraction influences greatly for the release of the bound phenolics which are trapped or conjugated in the cell walls. Accordingly, it was revealed that the bound phenolic fraction consists of a distinct fraction of the total phenolic content (Figure 1). Therefore, inclusion of the bound phenolics in studies related to quantification and evaluation of antioxidant activity of cereals is essential. Otherwise, it would result in underestimation during the evaluation of phenolic content if the bound portion is not considered. 


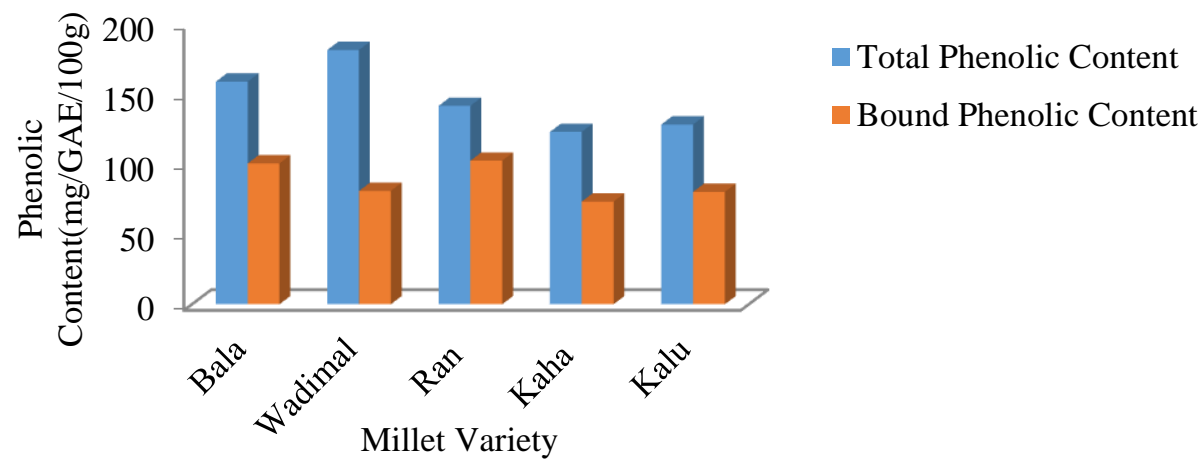

Figure 1. Total and bound phenolic contents of millet varieties

\section{DPPH radical scavenging activity}

In the evaluation of radical scavenging components in food and biological materials, the DPPH radical scavenging assay is frequently employed as a screening test. Figure 2 shows the $\mathrm{IC}_{50}$ value of millet extracts. According to the study, all the millet varieties exhibited strong antioxidant activities. The strongest radical scavenging activity was exhibited by Wadimal variety of finger millet and the least by the Kalu variety of foxtail millet.

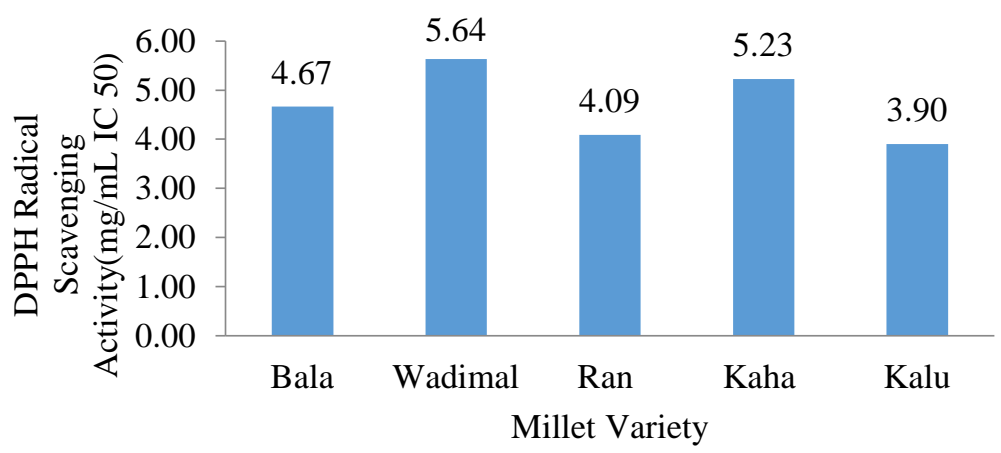

Figure 2. DPPH Radical Scavenging Activity of different millet varieties

\section{Ferric reducing antioxidant power (FRAP) assay}

According to the Figure 3, Bala Kurakkan exhibited the highest antioxidant activity as determined in the FRAP assay $(58.18 \mathrm{mg} / 100 \mathrm{~g} / \mathrm{GAE})$. Three varieties of foxtail millet have showed less FRAP activity than the finger millets. 


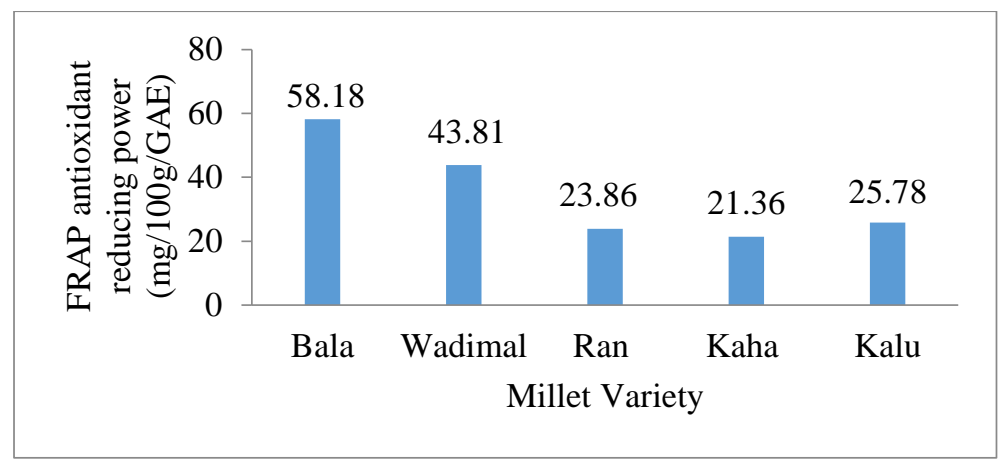

\section{Figure 3.Ferric reducing antioxidant power (FRAP) of different millet verities}

Total phenolic content of all the millet varieties strongly correlated with their antioxidant activities determined by other two methods namely FRAP and DPPH. The correlation coefficients calculated were as follows: TPC versus FRAP $=0.750$; TPC versus DPPH, $=-$ 0.824 but the correlation between FRAP and DPPH was reported to be moderately correlated which shows a value of 0.543 . According to the present study the bound fraction was significantly $(P<0.05)$ higher than the TPC. However, a variation was identified in the trend between soluble and bound phenolic fractions of different millet varieties (Figure 1). The correlation between the bound fraction and the TPC was found to be 0.25 . But there are studies which report that the amount of bound phenolic fraction is lesser than that of their free counterparts (Hung et al., 2008). The extraction method employed, temperature, $\mathrm{pH}$ and even the ratio between the solvent to the sample can affect the results generated. Since the present study was assisted with an ultrasonic extraction procedure, it can be suggested as an alternative for the conventional methods.

\section{CONCLUSIONS}

The present study demonstrated a wide variation in the polyphenolic content (PC) and antioxidant activities (AOA) in the investigated millets widely grown in Sri Lanka. Among them, it was significant that Finger millet varieties showed higher antioxidant activities compared to the Foxtail varieties in all the assays performed. But the highest content of bound phenolic fraction was exhibited in Ran variety of Foxtail millet. Therefore, the results generated in the present study suggest that both the foxtail millet varieties (Wadimal and Bala) are a rich source of potent antioxidants.

\section{ACKNOWLEDGEMENTS}

The authors express their gratitude to FAO (Food and Agriculture Organization) and UNEP (Unites Nations Environment Programme) for funding and Plant Genetic Resource Center (PGRC), Department of Agriculture, Sri Lanka for assisting during the sample collection 


\section{REFERENCES}

Chandrasekara, A. and Shahidi, F. (2010). Inhibitory activities of soluble and bound millet seed phenolics on free radicals and reactive oxygen species. J. Agric. Food Chem. 59(1), 428-436.

Chandrasekara, A. and Shahidi, F. (2010). Content of insoluble bound phenolics in millets and their contribution to antioxidant capacity. J. Agric. Food Chem. 58(11), 6706-6714.

Chandrasekara, A., Naczk, M. and Shahidi, F. (2012). Effect of processing on the antioxidant activity of millet grains. Food Chem. 133(1), 1-9.

Hegde, P.S., Rajasekaran, N.S. and Chandra, T.S. (2005). Effects of the antioxidant properties of millet species on oxidative stress and glycemic status in alloxan-induced rats. Nutr. Res. 25(12), 1109-1120.

McKeown, N.M., Meigs, J.B., Liu, S., Wilson, P.W. and Jacques, P.F. (2002). Whole-grain intake is favorably associated with metabolic risk factors for type 2 diabetes and cardiovascular disease in the Framingham Offspring Study. Am. J. Clin. Nutr. 76(2), 390398.

Reihani, S.F.S. and Azhar, M.E. (2012). Antioxidant activity and total phenolic content in aqueous extracts of selected traditional Malaysia salad (ulam). Int. Food Res. J. 19(4), 14391444.

Rupasinghe, H.V., Wang, L., Huber, G.M. and Pitts, N.L. (2008). Effect of baking on dietary fibre and phenolics of muffins incorporated with apple skin powder. Food Chem. 107(3), 1217-1224.

Singleton, V.L. and Rossi, J.A. (1965). Colorimetry of total phenolics with phosphomolybdic-phosphotungstic acid reagents. Am. J. Enology Vitic. 16(3), 144-158.

Thompson, L.U. (1993). Potential health benefits and problems associated with antinutrients in foods. Food Res. Int. 26(2), 131-149.

Van Hung, P. and Morita, N. (2008). Distribution of phenolic compounds in the graded flours milled from whole buckwheat grains and their antioxidant capacities. Food chem. 109(2), 325-331. 